\title{
Profilaxis de enfermedad tromboembólica en pacientes hospitalizados con patología médica, estrechando la brecha entre las guías y la práctica clínica
}

\author{
ANDRÉS AIZMAN, EDUARDO ABBOTT, LUIS ROJAS
}

Departamento de

Medicina Interna. Escuela de Medicina. Facultad de Medicina. Pontificia Universidad Católica de Chile.

Recibido el 23 de junio de 2010, aceptado el 25 de abril de 2011.

Correspondencia a: Andrés Aizman Sevilla

Marcoleta $367.5^{\circ}$ piso.

Servicio de Medicina.

Fono: oficina: 02-3543151,

Fax: 02- 6394985

E-mail: andresaizman@ gmail.com

\section{Strategies for the prophylaxis of thromboembolic disease among medical patients}

Thromboembolic disease is the main preventable cause of in-hospital death. Approximately 10\% of nosocomial deaths are attributable to pulmonary embolism and in most cases, the diagnosis is not suspected before the autopsy. There are cost effective measures to decrease the incidence of thromboembolic disease. Pharmacological prophylaxis decreases the incidence of deep venous thrombosis by $65 \%$ and the incidence of pulmonary embolism by 35 to 55\%. Despite this data and the presence of clinical guidelines, prophylaxis of thromboembolic disease is used only in $40 \%$ of medical patients and in 65\% of surgical patients with recommended indications. We review the evidence that supports the use of thromboprophylaxis and the different strategies that may increase the compliance of physicians with its use. A protocol implemented in our institution is also proposed.

(Rev Med Chile 2011; 139: 1210-1217).

Key words: Anticoagulants; Pulmonary embolism; Venous thromboembolism.
L

a enfermedad tromboembólica (ETE), constituida por la trombosis venosa profunda (TVP) y el tromboembolismo pulmonar (TEP), tiene una incidencia 100 a 130 veces mayor en pacientes hospitalizados en comparación con la población general ${ }^{1}$. Alrededor de $25 \%$ de todos los eventos ocurre durante o tiene relación con una hospitalización reciente. Pese a que históricamente ha sido un tema abordado y estudiado en pacientes quirúrgicos, entre 70 y $80 \%$ de los TEP y 50 y $70 \%$ de los eventos de TVP sintomáticos asociados a hospitalización ocurren en pacientes no quirúrgicos ${ }^{2,3}$.

El TEP es responsable de aproximadamente $10 \%$ de las muertes dentro del hospital, y en la mayoría de estos casos (más de 70\%) el diagnóstico nunca fue sospechado clínicamente. De esta manera, la enfermedad tromboembólica se ha transformado en la principal causa de muerte prevenible dentro del hospital ${ }^{4-6}$.
Cincuenta a 80\% de los casos de TVP son asintomáticos, y en aproximadamente el 50\% de los pacientes con TVP proximal coexisten trombosis pulmonares asintomáticas ${ }^{7}$. Esto podría explicar el subdiagnóstico de la enfermedad y probablemente la subutilización de profilaxis tromboembólica en pacientes hospitalizados.

La importancia de la enfermedad tromboembólica radica no sólo en su frecuencia, sino también en sus complicaciones agudas y crónicas. Dentro de estas últimas destacan el síndrome postflebítico y la hipertensión pulmonar crónica, situaciones que generan una importante discapacidad y elevados costos para los sistemas de salud.

Además de la inmovilización, la edad avanzada y numerosas comorbilidades hacen que un porcentaje significativo de los pacientes médicos hospitalizados posean un alto riesgo de desarrollar complicaciones tromboembólicas. En estudios prospectivos, la incidencia de TVP en pacientes 
que no reciben profilaxis puede llegar a $15 \%$ y la de TEP a $1,5 \%{ }^{8,9}$.

Diversos estudios han demostrado que la profilaxis farmacológica con heparinas es segura y eficaz, logrando reducciones del riesgo relativo de TVP y TEP de 40 a $70 \%^{8-10}$, constituyendo además una medida costo-efectiva ${ }^{1}$.

A pesar de lo anterior, un porcentaje reducido de los pacientes médicos con riesgo reciben profilaxis adecuada ${ }^{2}$. En el estudio multinacional ENDORSE, sólo 39,5\% de 37.356 pacientes médicos recibía profilaxis tromboembólica teniendo indicación. Esto contrastaba con el 58,5\% en población quirúrgica ${ }^{10}$.

La implementación de estrategias locales que faciliten el uso sistemático de tromboprofilaxis en pacientes médicos, contribuirá a disminuir en forma significativa la cantidad de eventos tromboembólicos asociados a hospitalización.

\section{Estratificando el riesgo}

En el siglo XIX Virchow planteó que el éstasis venoso, la injuria endotelial y la hipercoagula- bilidad eran los factores de riesgo asociados al desarrollo de trombosis. Hoy esta triada sigue teniendo vigencia, estando sus elementos presentes en los distintos factores de riesgo que han ido identificándose con posterioridad. Los factores de riesgos más frecuentes se describen en la Tabla 1.

La estimación del riesgo de cada paciente es fundamental al momento de indicar una intervención que sea costo-efectiva. Si bien algunas herramientas han sido diseñadas para estimar el riesgo en forma individual, ninguna de éstas ha sido validada en forma prospectiva. Así, las recomendaciones de profilaxis en pacientes médicos siguen siendo poco específicas.

A pesar de lo anterior, es importante destacar que $75-80 \%$ de los pacientes médicos hospitalizados posee al menos un factor de riesgo ${ }^{11} \mathrm{y}$ un porcentaje importante de ellos tiene 3 o más factores de riesgo en forma simultánea ${ }^{1,12}$.

La edad en pacientes hospitalizados constituye sin duda uno de los principales factores de riesgo de ETE. La incidencia aumenta de aproximadamente 10 en 100.000 individuos/año en la población general a 300-500 eventos por 100.000 individuos/año en aquellos mayores de 70 años ${ }^{13}$.

Tabla 1. Principales factores de riesgo para enfermedad tromboembólica

\begin{tabular}{|ll|}
\hline Factores relacionados con el paciente & Factores relacionados a enfermedad \\
\hline Edad & Insuficiencia cardiaca - IAM \\
\hline Obesidad & Insuficiencia respiratoria crónica \\
\hline Paresia-Plejia extremidades inferiores & Inmovilización \\
\hline Várices-Insuficiencia venosa & Embarazo-puerperio \\
\hline Trombofilias primarias & Infección severa \\
\hline Historia previa de ETE (personal/familiar) & Cirugía \\
\hline & Trauma mayor \\
& Enfermedad reumatológica activa \\
\hline Enfermedad inflamatoria intestinal \\
\hline Cáncer-terapia anticancerosa \\
\hline Estrógenos \\
Síndrome nefrótico \\
\hline Accidente vascular encefálico (AVE) \\
Ingreso a cuidados Intensivos \\
Catéter venoso central (CVC) \\
Trastornos mieloproliferativos \\
\hline
\end{tabular}


Sobre esto se suman una serie de otros factores propios del paciente (insuficiencia cardiaca, cáncer, infecciones severas u otros) y de la hospitalización (inmovilización prolongada, procedimientos invasivos, etc).

Especial mención ameritan algunas patologías como el cáncer, que aumenta el riesgo en al menos 6 veces. El riesgo es particularmente alto en pacientes con tumores cerebrales y adenocarcinomas (páncreas, pulmón, ovario, riñón, colon, estómago y próstata). En globo, se estima que el cáncer es responsable de $20 \%$ de todos los eventos tromboembólicos $^{14}$. La quimioterapia y la hormonoterapia también pueden aumentar significativamente el riesgo de $\mathrm{ETE}^{15-17}$.

Otra situación que merece atención es la de pacientes con accidente vascular encefálico (AVE). La incidencia de TVP en pacientes con AVE y hemiplejia sin tromboprofilaxis puede alcanzar hasta $75 \%{ }^{18}$, porcentaje similar al experimentado por pacientes operados de fractura de cadera. El TEP por su parte, es responsable de hasta $25 \%$ de las muertes precoces después de un $\mathrm{AVE}^{19}$.

En suma, en ocasiones es difícil estimar el riesgo de desarrollar patología tromboembólica en el paciente individual y no existen herramientas validadas que faciliten hacerlo. Sin embargo, cada vez se conocen más y mejor los factores de riesgo. El Colegio Americano de Médicos del Tórax recomienda que pacientes hospitalizados por más de 3 días, mayores de 40 años y que posean algún otro factor de riesgo, sean considerados candidatos para tromboprofilaxis ${ }^{1}$.

\section{Profilaxis, alternativas y estudios clínicos}

Existen distintas alternativas de tromboprofilaxis. Éstas pueden dividirse en medidas mecánicas y farmacológicas.

\section{Profilaxis mecánica o no farmacológica}

La deambulación mejora el flujo venoso y es ampliamente recomendada en población quirúrgica. Sin embargo, su implementación en población médica está restringida a un bajo porcentaje del total de pacientes, tanto por condiciones del enfermo como del funcionamiento hospitalario.

Por otra parte, existe poca información que permita estimar la utilidad de las medias y dispositivos de compresión intermitente en pacientes médicos. No existen grandes estudios que permitan demostrar su utilidad en este tipo de pacientes.

El estudio publicado más grande en población médica, el CLOTS 1, reclutó a más de 2.500 pacientes con AVE isquémico y no logró demostrar beneficios en el grupo asignado en forma aleatoria a medias compresivas, es más, este grupo presentó más complicaciones como úlceras y necrosis cutánea ${ }^{20}$.

Las medias de compresión graduada y los dispositivos de compresión intermitente sí han demostrado disminuir la incidencia de eventos tromboembólicos en población quirúrgica en al menos 50\%. Su beneficio es evidente cuando se comparan contra placebo, pero también se mantiene en pacientes que están bajo profilaxis farmacológica ${ }^{21}$.

Extrapolando datos obtenidos en población quirúrgica, y tomando en cuenta el perfil de seguridad de este tipo de intervención, la profilaxis mecánica parece ser una buena alternativa para pacientes de alto riesgo hemorrágico, como aquellos con hemorragia digestiva reciente o trastornos de la coagulación (trombocitopenia severa por ejemplo). También pudiera considerarse su uso en forma combinada con medidas farmacológicas en pacientes de muy alto riesgo tromboembólico.

La mala adherencia de los pacientes y personal de salud en el uso de medias y dispositivos de compresión intermitente constituyen un elemento a considerar al indicar e implementar este tipo de intervenciones.

\section{Profilaxis farmacológica}

Existen distintas alternativas de profilaxis farmacológica; heparina no fraccionada (HNF), heparinas de bajo peso molecular (HBPM), fundaparina y los nuevos anticoagulantes, rivaroxaban y dabigatran.

La evidencia demuestra en forma clara que la profilaxis farmacológica disminuye la incidencia de eventos tromboembólicos en pacientes médicos. Dos metaanálisis recientes, demuestran que el uso de profilaxis farmacológica disminuye en aproximadamente $65 \%$ el riesgo relativo de TVP y entre 35 y $55 \%$ el de TEP (62\% para TEP fatal $)^{22,23}$. Esto se asocia a un aumento no significativo de episodios hemorrágicos mayores.

La reducción del riesgo absoluto para TEP y TEP fatal fue sin embargo modesta, 0,29 y 0,25\%, respectivamente en el metaanálisis de Dentali ${ }^{22}$. 
Por ende, 345 pacientes médicos hospitalizados en riesgo deberían recibir profilaxis para prevenir un TEP sintomático y 400 para prevenir un TEP fatal.

Las contraindicaciones de tromboprofilaxis se expresan en la Tabla 2.

\section{Heparina no fraccionada versus placebo}

El metaanálisis de Wein mostró que el uso de HNF disminuye el riesgo relativo de TVP en $67 \%$ y el de TEP en $36 \%$. No hubo diferencias de mortalidad respecto a placebo ${ }^{23}$. El uso de HNF en dosis de 5.000 UI tres veces al día versus dos veces al día se asoció a una mayor reducción del riesgo de TVP ( 73 vs $48 \%$ ), hallazgo similar al publicado en el metaanálisis de King ${ }^{24}$, en el cual hubo una tendencia no significativa a reducir la tasa de TVP (5,4 vs 3,5 eventos por cada 1.000 paciente-día) y TEP ( 1,5 vs 0,5$)$ a expensas de una mayor tasa de sangrados mayores $(0,33$ vs 0,73$)$. En base a estos resultados podría plantearse el uso de HNF tres veces al día en pacientes de alto riesgo tromboembólico y dos veces al día en aquellos con riesgo bajo-moderado o mayor riesgo hemorragíparo.

\section{Heparinas de bajo peso molecular versus placebo}

El metaanálisis de Wein mostró que el uso de HBPM confiere una disminución del riesgo de TVP de $44 \%$ y de TEP de $63 \%$ asociado a un aumento no significativo de eventos hemorrágicos mayores. Tampoco hubo reducción en la mortalidad $^{23}$.

La información del beneficio de las HBPM proviene principalmente de dos estudios randomizados: MEDENOX y PREVENT.

El estudio MEDENOX comparó dos dosis de enoxaparina (20 y $40 \mathrm{mg}$ al día) contra placebo en 1.102 pacientes médicos tratados en promedio por 7 días. El grupo asignado a $40 \mathrm{mg}$ tuvo una reducción significativa del riesgo relativo de eventos tromboembólicos (TVP y TEP) de 63\% $(5,5$ vs $14,9 \%)$. No hubo diferencias significativas en mortalidad ni complicaciones hemorrágicas. El grupo asignado a $20 \mathrm{mg}$ no tuvo diferencias con el placebo ${ }^{8}$.

El estudio PREVENT distribuyó en forma aleatoria a 3.706 pacientes médicos a recibir dalteparina $5.000 \mathrm{U}$ al día por 14 días o placebo. El grupo asignado a dalteparina también presentó una reducción significativa en el riesgo relativo de sufrir un evento tromboembólico de $44 \%$ (2,8 vs
Tabla 2. Contraindicaciones de profilaxis farmacológica

Sangrado activo o alto riesgo de hemorragia

Recuento de plaquetas $<100.00$

Historia de trombocitopenia inducida por heparina (HIT)

Cirugía SNC reciente

AVE hemorrágico (hasta el $3^{\circ}$ día)

HTA severa no controlada

Hipersensibilidad al medicamento

12 hrs previo a punción lumbar (sólo HBPM)

5,0\%). Tampoco hubo diferencias en mortalidad y eventos hemorrágicos ${ }^{25}$.

Un subanálisis de este estudio en pacientes obesos y ancianos demostró que el beneficio y la seguridad se mantenía en distintos rangos de peso (salvo en aquellos pacientes con IMC mayor a 40) y en aquellos mayores de 75 años.

\section{Fundaparina versus placebo}

Existe un solo estudio de fundaparina en pacientes médicos (ARTEMIS). En dicho estudio 849 pacientes médicos similares a los incluidos en los estudios anteriores fueron distribuidos en forma aleatoria a fundaparina $2,5 \mathrm{mg}$ al día por 6 a 14 días o placebo. El grupo asignado a fundaparina tuvo una reducción de $47 \%$ en el riesgo relativo de presentar eventos tromboembólicos (5,6 versus $10,5 \%)^{9}$.

\section{Heparinas de bajo peso molecular versus heparina no fraccionada}

Existe más de un metaanálisis comparando la efectividad y seguridad de las HBPM y la $\mathrm{HNF}^{23,26-28}$. En general, muestran resultados similares entre sí aunque no del todo concluyentes. En el metaanálisis de Wein, las HBPM producían una mayor reducción del riesgo de TVP (32\%) y una tendencia no significativa a reducir el riesgo de TEP y complicaciones hemorrágicas. No hubo diferencias en términos de mortalidad ${ }^{24}$.

En el metaanálisis previo de Mismetti ${ }^{27}$, no hubo diferencias significativas entre HBPM y HNF en prevención de eventos tromboembólicos pero sí una disminución significativa en el riesgo de hemorragias mayores (52\%).

El metaanálisis más reciente de $\mathrm{Bump}^{28}$ no mostró diferencias significativas entre HBPM y 
HNF en términos de reducción del riesgo de TVP o TEP, aunque sí una tendencia no significativa a presentar menos eventos hemorrágicos a favor de las HBPM.

En poblaciones de riesgo elevado, 2 estudios aleatorios muestran una mayor efectividad de HBPM por sobre HNF: PREVAIL y PRINCE.

El estudio PREVAIL estudió con distribución aleatoria 1.762 pacientes con AVE isquémico incapaces de deambular a enoxaparina $40 \mathrm{mg}$ al día o HNF 5.000 UI 2 veces al día (esquema discutible, pero el más utilizado según los autores) por 6 a 14 días. El grupo asignado a enoxaparina tuvo un riesgo relativo $43 \%$ menor de presentar un evento tromboembólico (10 vs 18\%). No hubo diferencias en la incidencia de complicaciones hemorrágicas, incluyendo hemorragias intracraneanas ${ }^{29}$.

El estudio PRINCE estudió con distribución aleatoria 665 pacientes hospitalizados por insuficiencia cardiaca congestiva (Capacidad Funcional III/IV) o enfermedad respiratoria grave a recibir enoxaparina $40 \mathrm{mg}$ al día o HNF 5.000 UI cada 8 horas. La heparina de bajo peso fue más efectiva reduciendo el riesgo de TVP $(9,7 \% \text { versus } 16,1 \%)^{30}$.

En suma, las HBPM son al menos tan efectivas como la HNF y parecen ser más seguras. Probablemente la comodidad de administración tanto para el paciente como para el personal de enfermería, hacen más atractivo su uso por sobre la HNF.

\section{Otras alternativas}

\section{Aspirina}

Si bien el ácido acetilsalicílico pudiera tener un efecto discreto disminuyendo la incidencia de eventos tromboembólicos ${ }^{31}$, su uso, así como el de otros antiinflamatorios no esteroidales o antiagregantes plaquetarios, no está recomendado como medida de tromboprofilaxis ${ }^{1}$.

\section{Nuevos antitrombóticos orales}

Especial interés ha surgido en el uso de nuevos antitrombóticos orales como rivaroxaban (inhibidor del factor Xa) y dabigatran (inhibidor directo de la trombina), tras la experiencia en estudios de profilaxis de TVP en pacientes traumatológicos. Ambos fármacos han sido aprobados para su uso en pacientes postoperados de prótesis de cadera $y$ rodilla ${ }^{32-35}$.

No existe aún evidencia para su uso en pacien- tes médicos, sin embargo, se encuentra en curso al menos un estudio para rivaroxaban (estudio MAGELLAN, NCT00571649) que debiera arrojar resultados sobre su utilidad en un futuro cercano.

\section{Pacientes con insuficiencia renal}

No existen trabajos que evalúen el beneficio de profilaxis farmacológica en el contexto de insuficiencia renal crónica. Es importante recordar que las heparinas de bajo peso molecular, a diferencia de la heparina no fraccionada que se metaboliza en el sistema retículo-endotelial, se excretan por la orina. En estos pacientes parece aconsejable utilizar heparina no fraccionada cada 8 ó $12 \mathrm{hrs}$ según el riesgo individual.

\section{Estrategias para mejorar la indicación de profilaxis en el hospital}

Como ya se ha comentado, la profilaxis de enfermedad tromboembólica sigue siendo subindicada ${ }^{11,37,38}$ pese a la evidencia que la respalda y a la existencia de guías clínicas que recomiendan su uso ${ }^{1,39}$.

La subutilización está relacionada a una serie de factores. Entre ellos destaca una baja percepción respecto a la magnitud del problema y del beneficio de la profilaxis ${ }^{40}$. Por otro lado, la evaluación y estratificación del riesgo en pacientes médicos es más compleja que en pacientes quirúrgicos (donde se utiliza el tipo de cirugía como principal indicador). Por último, el temor a complicaciones hemorrágicas sigue siendo una barrera importante, especialmente en algunas patologías como el AVE. Dicho temor se basa en una sobreestimación del riesgo hemorragíparo asociado a dosis bajas de anticoagulantes.

Métodos pasivos, como material educativo, charlas y probablemente este mismo artículo, tienen poco impacto al momento de mejorar las prácticas de tromboprofilaxis. El establecimiento de protocolos, registros de actividad y su seguimiento han demostrado mejorar la indicación en términos cuantitativos y cualitativos. Por ejemplo, en el trabajo de Kucher et $\mathrm{al}^{41}$, el uso de alertas asociadas a la ficha electrónica de cada paciente permitió aumentar el uso de profilaxis tanto farmacológica (23,6 vs $13 \%$ ) como mecánica (10 vs $1,5 \%)$. Esto se asoció a una reducción significativa de $41 \%$ de presentar algún evento tromboembólico a 3 meses ( 4,9 vs $8,2 \%)$. De hecho, la guía del $\mathrm{ACCP}^{1}$ recomienda el diseño de una estrategia 
Paciente $>40$ años hospitalizado con uno o más factores de riesgo de evento trombótico

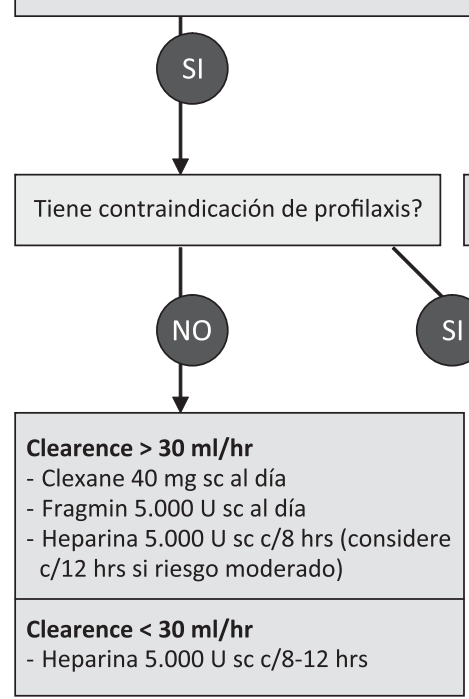

Considere uso de heparinas más medias en pacientes de alto riesgo Considere su uso en < de 40 años con alto riesgo

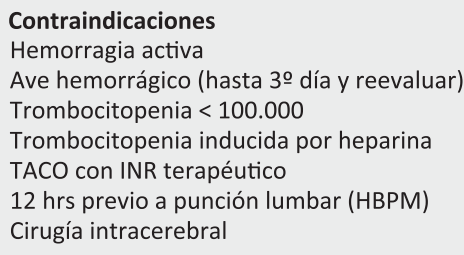

Trombocitopenia inducida por heparina

TACO con INR terapéutico

12 hrs previo a punción lumbar (HBPM)

Cirugía intracerebral

Figura 1. Protocolo de Profilaxis de Enfermedad Tromboembólica en pacientes médicos. Servicio de Medicina Interna, Hospital Clínico UC.

activa en cada hospital, establecida como política formal para toda la institución ${ }^{40,41}$.

En nuestro servicio de medicina interna hemos implementado un protocolo de profilaxis de enfermedad tromboembólica buscando optimizar el uso correcto de la profilaxis farmacológica y mecánica en nuestros pacientes (Figura 1).

\section{Conclusiones}

La enfermedad tromboembólica es un problema frecuente en pacientes médicos hospitalizados. Ha sido catalogada como la principal causa de muerte prevenible en dichos pacientes. A pesar de existir suficiente evidencia demostrando el beneficio de la profilaxis farmacológica, ésta sigue siendo una medida subutilizada. El implementar estrategias activas para mejorar la indicación de profilaxis en nuestros hospitales permitirá reducir el gran número de eventos tromboembólicos, disminuyendo morbilidad y mejorando la calidad de vida de nuestros pacientes.

\section{Referencias}

1. Geerts WH, Bergqvist D, Pineo GF, Heit JA, Samama $\mathrm{CM}$, Lassen MR, et al. Prevention of venous thromboembolism: ACCP evidence-based clinical practice guidelines (8th edition). Chest 2008; 133 (suppl 6): 381s-453s.

2. Goldhaber SZ, Tapson VF. A prospective registry of 5,451 patients with ultrasound-confirmed deep vein thrombo- 
sis. Am J Cardiol 2004; 93: 259-62.

3. Anderson FA Jr, Wheeler HB, Goldberg RJ, Hosmer DW, Patwardhan NA, Javanovic B, et al. A population-based perspective of the hospital incidence and case-fatality rates of deep vein thrombosis and pulmonary embolism: the Worcester DVT Study. Arch Intern Med 1991; 151: 933-8.

4. Baglin TP, White K, Charles A. Fatal pulmonary embolism in hospitalised medical patients. J Clin Pathol 1997; 50: 609-10.

5. Sandler DA, Martin JF. Autopsy proven pulmonary embolism in hospital patients: are we detecting enough deep vein thrombosis? J R Soc Med 1989; 82: 203-5.

6. Stein PD, Henry JW. Prevalence of acute pulmonary embolism among patients in a general hospital and at autopsy. Chest 1995; 108: 978-81.

7. Meignan M, Rosso J, Gauthier H, Brunengo F, Claudel S, Sagnard L, et al. Systematic lung scans reveal a high frequency of silent pulmonary embolism in patients with proximal deep venous thrombosis. Arch Intern Med 2000; 160: 159-64.

8. Samama MM, Cohen AT, Darmon J-Y, Desjardins L, Eldor A, Janbon C, et al. A comparison of enoxaparin with placebo for the prevention of venous thromboembolism in acutely ill medical patients. N Engl J Med 1999; 341: 793-800.

9. Cohen AT, Davidson BL, Gallus AS, Lassen MR, Prins $\mathrm{MH}$, Tomkowski W, et al. Efficacy and safety of fondaparinux for the prevention of venous thromboembolism in older acute medical patients: randomized placebo controlled trial. BMJ 2006; 332: 325-9.

10. Cohen AT, Tapson VF, Bergmann JF, Goldhaber SZ, Kakkar AK, Deslandes B, et al. Venous thromboembolism risk and prophylaxis in the acute hospital care setting (ENDORSE study): a multinational cross-sectional study. Lancet 2008; 371: 387-94.

11. Anderson Jr FA, Decousus H, Bergmann JF, Chong S, Froehlich J, Johnson J, et al. A multinational observational cohort study in hospitalized medical patients of practices in prevention of venous thromboembolism and clinical outcomes: findings of the International Medical Prevention Registry of Venous Thromboembolism (IMPROVE). J Thromb Haemost 2003; 1 (Suppl 1): P1438.

12. Sobieraj DM. Development and implementation of a program to assess medical patients' need for venous thromboembolism prophylaxis. Am J Health Syst Pharm 2008; 65: 1755-60.

13. White RH. The epidemiology of venous thromboembolism. Circulation 2003; 107 (suppl 1): 14-8.

14. Prandoni P, Falanga A, Piccioli A. Cancer and venous thromboembolism. Lancet Oncol 2005; 6: 401-10.

15. Otten HM, Mathijssen J, Ten Cate H, Soesan M, Inghels $\mathrm{M}$, Richel DJ, et al. Symptomatic venous thromboembolism in cancer patients treated with chemotherapy: an underestimated phenomenon. Arch Intern Med 2004; 164: 190-4.

16. Fisher B, Costantino JP, Wickerham DL, Redmond CK, Kavanah M, Cronin WM, et al. Tamoxifen for prevention of breast cancer: report of the National Surgical Adjuvant Breast and Bowel Project P-1 Study. J Natl Cancer Inst 1998; 90: 1371-88.

17. Hernández RK, Sorensen HT, Pedersen L, Jacobsen J, Lash TL. Tamoxifen treatment and risk of deep venous thrombosis and pulmonary embolism: a Danish population-based cohort study. Cancer 2009; 115: 4442-9.

18. Kamphuisen PW, Agnelli G, Sebastianelli M. Prevention of venous thromboembolism after acute ischemic stroke.J Thromb Haemost 2005; 3: 1187-94.

19. Kelly J, Rudd A, Lewis R, Hunt BJ. Venous thromboembolism after acute stroke. Stroke 2001; 32: 262-7.

20. Dennis M, Sandercock PA, Reid J, Graham C, Murray G, Venables $\mathrm{G}$, et al. Effectiveness of thigh-length graduated compression stockings to reduce the risk of deep vein thrombosis after stroke (CLOTS trial 1): a multicentre, randomised controlled trial. Lancet 2009; 373: 1958-65.

21. Amaragiri SV, Lees TA. Elastic compression stockings for prevention of deep vein Thrombosis. Cochrane Database Syst Rev 2000; CD001484.

22. Dentali F, Douketis JD, Gianni M, Lim W, Crowther MA. Meta-analysis: anticoagulant prophylaxis to prevent symptomatic venous thromboembolism in hospitalized medical patients. Ann Intern Med 2007; 146: 278-88.

23. Wein L, Wein S, Haas SJ, Shaw J, Krum H. Pharmacological Venous Thromboembolism Prophylaxis in Hospitalized Medical Patients: A Meta-analysis of Randomized Controlled Trials. Arch Intern Med 2007; 167: 1476-86.

24. King CS, Holley AB, Jackson JL, Shorr AF, Moores LK. Twice vs three times daily heparin dosing for thromboembolism prophylaxis in the general medical population: A metaanalysis. Chest 2007; 131: 507-16.

25. Leizorovicz A, Cohen AT, Turpie AG, Olsson CG, Vaitkus PT, Goldhaber SZ. Randomized, placebo-controlled trial of dalteparin for the prevention of venous thromboembolism in acutely ill medical patients. Circulation 2004; 110: 874-9.

26. Kanaan AO, Silva MA, Donovan JL, Roy T, Al-Homsi AS. Meta-analysis of venous thromboembolism prophylaxis in medically ill patients. Clin Ther 2007; 29: 2395-405.

27. Mismetti P, Laporte-Simitsidis S, Tardy B, Cucherat M, Buchmüller A, Juillard-Delsart D, et al. Prevention of venous thromboembolism in internal medicine with 
unfractionated or low-molecular-weight heparins: a meta-analysis of randomised clinical trials. Thromb Haemost 2000; 83: 14-9.

28. Bump GM, Dandu M, Kaufman SR, Shojania KG, Flanders SA. How Complete Is the Evidence for Thromboembolism Prophylaxis in General Medicine Patientes? A Meta-Analysis of Randomized Controlled Trials. J Hosp Med 2009; 4: 289-97.

29. Sherman DG, Albers GW, Bladin C, Fieschi C, Gabbai AA, Kase CS, et al. The efficacy and safety of enoxaparin versus unfractionated heparin for the prevention of venous thromboembolism after acute ischaemic stroke (PREVAIL Study): an open-label randomised comparison. Lancet 2007; 369: 1347-55.

30. Kleber FX, Witt C, Vogel G, Koppenhagen K, Schomaker U, Flosbach CW. Randomized comparison of enoxaparin with unfractionated heparin for the prevention of venous thromboembolism in medical patients with heart failure or severe respiratory disease. Am Heart J 2003; 145: 614-21.

31. Karthikeyan G, Eikelboom JW, Turpie AG, Hirsh J. Does acetyl salicylic acid (ASA) have a role in the prevention of venous thromboembolism? Br J Haematol 2009 May 9.

32. Eriksson BI, Borris LC, Friedman RJ, Haas S, Huisman MV, Kakkar AK, et al. Rivaroxaban versus enoxaparin for thromboprophylaxis after hip arthroplasty. N Engl J Med 2008; 358: 2765-75.

33. Lassen MR, Ageno W, Borris LC, Lieberman JR, Rosencher N, Bandel TJ, et al. Rivaroxaban versus enoxaparin for thromboprophylaxis after total knee arthroplasty. $\mathrm{N}$ Engl J Med 2008; 358: 2776-86.

34. Eriksson BI, Dahl OE, Rosencher N, Kurth AA, van Dijk CN, Frostick SP, et al. Oral dabigatran etexilate vs subcutaneous enoxaparin for the prevention of venous thromboembolism after total knee replacement: the RE-
MODEL randomized trial. J Thromb Haemost 2007; 5 (11): 2178-85.

35. Eriksson BI, Dahl OE, Rosencher N, Kurth AA, van Dijk CN, Frostick SP, et al. Dabigatran etexilate versus enoxaparin for prevention of venous thromboembolism after total hip replacement: a randomised, double-blind, non-inferiority trial. Lancet 2007; 370: 949-56.

36. Monreal M, Kakkar AK, Caprini JA, Barba R, Uresandi $\mathrm{F}$, Valle $\mathrm{R}$, et al. The outcome after treatment of venous thromboembolism is different in surgical and acutely ill medical patients. Finding from the RIETE registry. J Thromb Haemost 2004; 2: 1892-8.

37. Goldhaber SZ, Tapson VF. A prospective registry of 5451 patients with ultrasound-confirmed deep vein thrombosis. Am J Cardiol 2004; 93: 259-62.

38. Nicolaides AN, Breddin HK, Fareed J, Goldhaber S, Haas S, Hull R, et al. Prevention of venous thromboembolism. International Consensus Statement. Guidelines compiled in accordance with the scientific evidence. Int Angiol 2001; 20 (1): 1-37.

39. Baglin T. Defining the population in need of thromboprophylaxis-making hospitals safer. Br J Haematol 2010; 149: 805-12.

40. Kucher N, Koo S, Quiroz R, Cooper JM, Paterno MD, Soukonnikov B, Goldhaber SZ. Electronic alerts to prevent venous thromboembolism among hospitalized patients. N Engl J Med 2005; 352: 969-77.

41. Timmons S, O'Callaghan C, O'Connor M, O'Mahony D, Twomey C. Audit guided action can improve the compliance with thromboembolic prophylaxis prescribing to hospitalized, acutely ill older adults. J Thromb Haemost 2005; 3: 2112-3.

42. Tooher R, Middleton P, Pham C, Fitridge R, Rowe S, Babidge W, et al. A systematic review of strategies to improve prophylaxis for venous thromboembolism in hospitals. Ann Surg 2005; 241: 397-415. 